\title{
Comparison of sputum induction and bronchoscopy in diagnosis of sputum smear-negative pulmonary tuberculosis: a systemic review and meta-analysis
}

Wen Luo ${ }^{1 \dagger}$, Yihua Lin ${ }^{1 \dagger}$, Zhibin Li , Wanyu Wang ${ }^{1}$ and Yonghong Shi ${ }^{1^{*}}$ (i)

\begin{abstract}
Background: Pulmonary tuberculosis is one of the most common infectious diseases worldwide. Patients with suspected pulmonary tuberculosis with negative smear are recommended to undergo further tests including sputum induction and bronchoscopy. Our study is aimed to compare sputum induction and bronchoscopic specimens in the diagnosis of sputum smear-negative pulmonary tuberculosis.
\end{abstract}

Method: PubMed, Web of Science, Cochrane Library and Embase were searched for eligible studies. The pooled sensitivities (SEN), specificities (SPE), positive likelihood ratio (PLR), negative likelihood ratio (NLR), diagnostic odds ratio (DOR) and 95\% confidence intervals (CI) were constructed, and the areas under the curves (AUCs) were calculated.

Results: Five studies with a total number of 586 cases were included. For mycobacterial culture, the SEN and SPE of sputum induction were $0.72(95 \% \mathrm{Cl}, 0.66-0.77)$ and $1.00(95 \% \mathrm{Cl}, 0.99-1.000)$ respectively, whereas the SEN and SPE of bronchoscopy were $0.70(95 \% \mathrm{Cl}, 0.64-0.75)$ and $1.00(95 \% \mathrm{Cl}, 0.99-1.00)$ respectively. Sputum induction had a similar AUC $(0.9564, \mathrm{SE}=0.0749)$ with bronchoscopy $(0.8618, \mathrm{SE}=0.1652)(P=0.602)$. For specimen of acid-fast bacilli smear, the SEN and SPE of sputum induction were $0.35(95 \% \mathrm{Cl}, 0.29-0.42)$ and $0.99(95 \% \mathrm{Cl}, 0.96-1.00)$ respectively, whereas the SEN and SPE of bronchoscopy were $0.38(95 \% \mathrm{Cl}, 0.32-0.45)$ and $0.99(95 \% \mathrm{Cl}, 0.96-1.00)$ respectively. There is no statistically significant difference in the AUC for sputum induction (0.6016) compared with bronchoscopy (0.8163) $(P=0.792)$.

Conclusions: For the diagnosis of sputum smear-negative pulmonary tuberculosis, the diagnosis yield of sputum induction and bronchoscopy is similar.

Keywords: Tuberculosis, Induced sputum, Bronchoscopy, Meta-analysis, Diagnosis

\footnotetext{
* Correspondence: shiyonghong_xmu@163.com

${ }^{+}$Wen Luo and Yihua Lin contributed equally to this work.

'Department of Pulmonary and Critical Care Medicine, the First Affiliated Hospital of Xiamen University, Xiamen 361001, China

Full list of author information is available at the end of the article
}

(c) The Author(s). 2020 Open Access This article is licensed under a Creative Commons Attribution 4.0 International License, which permits use, sharing, adaptation, distribution and reproduction in any medium or format, as long as you give appropriate credit to the original author(s) and the source, provide a link to the Creative Commons licence, and indicate if changes were made. The images or other third party material in this article are included in the article's Creative Commons licence, unless indicated otherwise in a credit line to the material. If material is not included in the article's Creative Commons licence and your intended use is not permitted by statutory regulation or exceeds the permitted use, you will need to obtain permission directly from the copyright holder. To view a copy of this licence, visit http://creativecommons.org/licenses/by/4.0/. The Creative Commons Public Domain Dedication waiver (http://creativecommons.org/publicdomain/zero/1.0/) applies to the data made available in this article, unless otherwise stated in a credit line to the data. 


\section{Background}

Pulmonary tuberculosis (TB) is one of the most common infectious disease worldwide and also one of the top 10 causes of death, especially in developing countries [1]. Early diagnosis is the most effective pulmonary tuberculosis control strategy because the early appropriate treatment renders these patients noninfectious and interrupts the chain of disease transmission. Acid-fast bacilli in sputum is recommended as the preliminary diagnostic method by the World Health Organization (WHO). However, the sensitivity of this method is low and the value in patients who cannot produce sputum spontaneously is very little $[2,3]$. It follows that the active respiratory specimens collection is an important strategy to early diagnosis of pulmonary tuberculosis [4].

Sputum induction and/or bronchoscopy are commonly used for the diagnosis in patients with suspected tuberculosis who do not produce sputum or have a negative acid-fast bacilli smear from spontaneous sputum. Sputum induction is a safe and effective method in obtaining specimens for acid-fast bacilli smear and mycobacterial culture [5]. In areas where bronchoscopy is not readily available, sputum induction offers an alternative or additional approach to the diagnosis of sputum smear-negative pulmonary tuberculosis [6]. Bronchoscopy is more invasive, more expensive, and lesstolerated than sputum induction, but bronchoscopy can provide specimens from the lesion area of the lung.

Over the past decade, several studies [5-9] have described the diagnosis yield of sputum induction in comparison with bronchoscopy in the sputum smearnegative pulmonary tuberculosis. Because of the heterogeneous populations and small sample sizes, the results of these studies were variable. This study is aimed to compare the sensitivity and specificity of sputum induction and bronchoscopy in the diagnosis of sputum smear-negative pulmonary tuberculosis by the method of meta-analysis [5-9].

\section{Methods}

This study was performed according to the Preferred Reporting Items for Systematic Reviews and Meta-Analyses (PRISMA) statement. The protocol for this meta-analysis is available in PROSPERO (CRD42019133766).

\section{Search for trials}

PubMed, Web of Science, Cochrane Library and Embase up to Mar 31, 2019 were searched by two investigators independently using search terms included "tuberculosis", "sputum induction", "induced sputum", "bronchoscopy" and "bronchoalveolar" to identify studies that met the inclusion criteria (see Additional file 1: Appendix S1, for complete search descriptions). There were no restrictions on language.

\section{Selection criteria}

Studies were selected based on the following inclusion criteria: (1) sputum induction and bronchoscopy were used to detect pulmonary tuberculosis in the same patient cohorts. The test result of induced-sputum specimens was the experimental group, whereas the test result of bronchoscopic specimens was regarded as the control group; (2) enough data to calculate the outcome data (true positive (TP), false positive (FP), true negative (TN), false negative $(\mathrm{FN})$ ); (3) the participants were diagnosed using the gold standard; (4) the gold standard for diagnosis of pulmonary tuberculosis [10] was defined in the study; (5) sputum induction and bronchoscopy had to be performed at the time of clinical presentation with suspected tuberculosis before administration of anti-tuberculosis therapy. The exclusion criteria were as follows: (1) the diagnostic method for tuberculosis did not include sputum induction and bronchoscopy; (2) reviews, case reports, letters, proceedings, or commentaries.

\section{Data extraction}

Two researchers independently extracted the following information from each study: name of study, first author, publication year, country, source of patients, the number of specimens collected, the concentrations of hypertonic saline, the type of nebulizers, the culture techniques, study type, sample size, reference standard, total number of $\mathrm{TB}$ diagnosis, type of bronchoscopic specimens, and outcome data (TP, FP, FN, and TN). Discrepancies were resolved by consensus.

\section{Risk-of-bias assessments}

Two researchers independently used the Quality Assessment of Diagnostic Accuracy Studies (QUADAS-2) tool, which was provided by RevMan (version 5.3, Cochrane Collaboration, Oxford, UK), to assess the risk of bias and applicability of diagnostic accuracy for the studies included. There were four sections in the QUADAS-2: patient selection, index test, reference standard, and flow and timing [11]. According to the following criteria, we judged the included studies as low risk, high risk or unclear bias: (1) if all the questions for a section were replied with "yes", then the risk of bias was judged as "low"; (2) if any question in a section was replied with "no", then risk of bias was judged as "high"; (3) when insufficient information was provided, the risk of bias was judged as "unclear bias". Meanwhile, we graded the applicability as low, high, or unclear with the above criteria.

\section{Statistical analysis}

We carried out a fixed-effects model to calculate pooled results and corresponding 95\% confidence intervals (CI) when there was no significant 
heterogeneity ( $P$ value of Cochran- $Q$ of $\mathrm{DOR}>0.1$ ); otherwise, we applied a random-effects model. Then we constructed the pooled sensitivities (SEN), specificities (SPE), positive likelihood ratio (PLR), negative likelihood ratio (NLR), diagnostic odds ratio (DOR), and $95 \%$ confidence intervals (CI) and afterwards calculated the areas under the curves (AUCs). We conducted $\mathrm{Z}$ tests to compare the diagnostic accuracies of sputum induction and bronchoscopic specimens directly. To calculate the degree of heterogeneity across studies, we selected the $I^{2}$ value and Q statistic of the chi-square test $(25-50 \%$, low heterogeneity; $51-75 \%$, medium heterogeneity; greater than $75 \%$, high heterogeneity) [12]. We used Deeks' funnel plot asymmetry test to assess the publication bias, and a $P$ value below 0.05 suggested the present of publication bias [13]. All statistical analyses were performed using Meta-DiSc Version 1.4, Review Manager Version 5.3, Stata Version 15 and R3.5.3 [14].

\section{Results}

Search results and study characteristics

The systematic literature searches identified 1809 potentially relevant studies. One thousand seven hundred and eighty two of these studies were eliminated before the full text assessment. The reasons of exclusion included duplicates, unrelated with the topic of the research, conference abstracts, reviews, case reports and letters. Twenty-seven records were screened in full-text articles and five qualified studies were included at last. The selection process was shown in Fig. 1. The five qualified studies [5-9] included a total of 586 cases. All the studies had sufficient data to quantitative synthesis for the mycobacterial culture of induced-sputum specimens and bronchoscopic specimens in the diagnosis of pulmonary tuberculosis, but only three studies had sufficient data on acid-fast bacilli smear [5-7]. Characteristics of those studies were presented in Table 1 . The quality of all the included studies, in terms of risk of bias and applicability concerns, was acceptable according to QUADAS-2 results (Fig. 2).

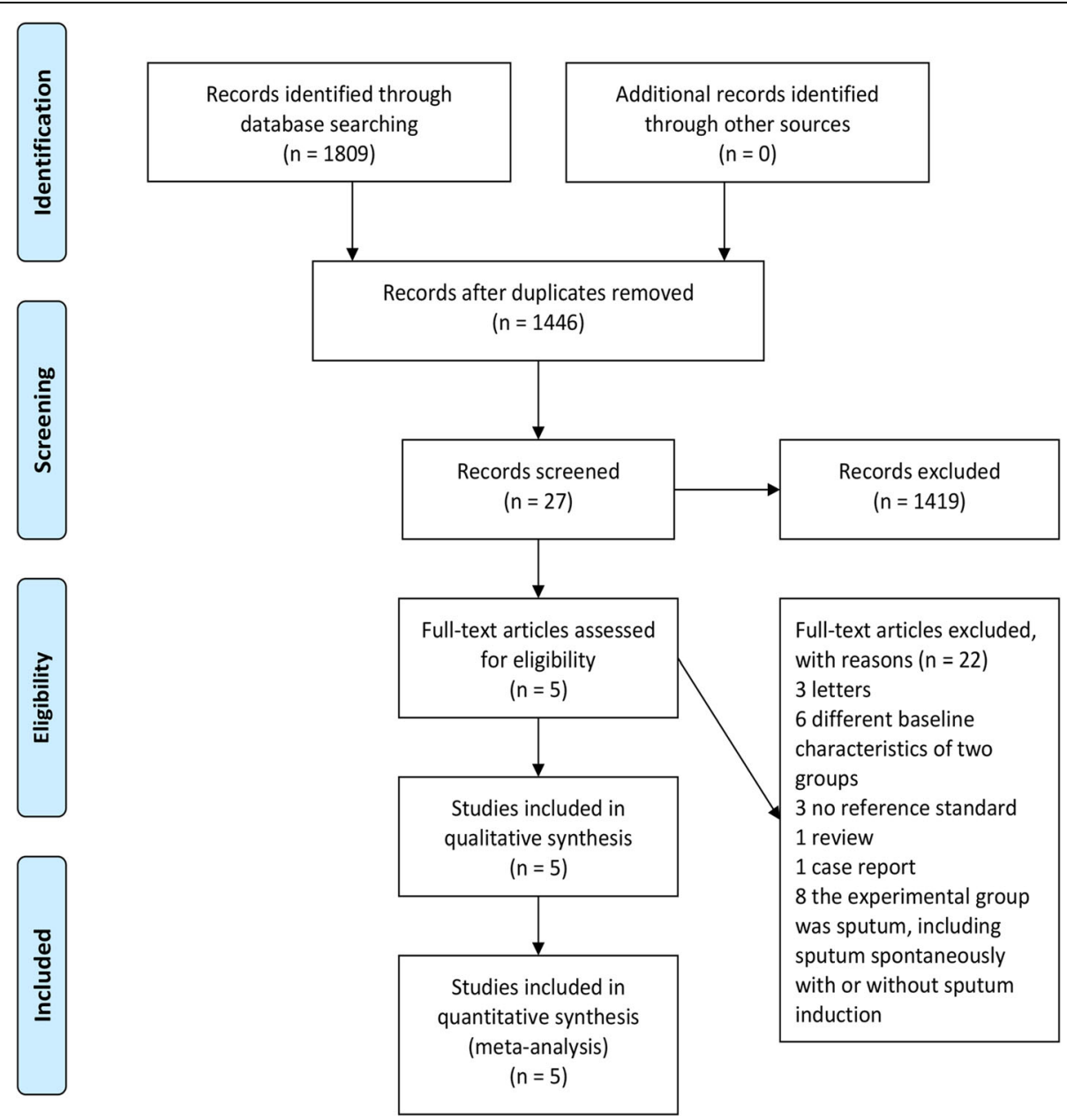

Fig. 1 Procedure used for selection of studies (PRISMA flow diagram) 


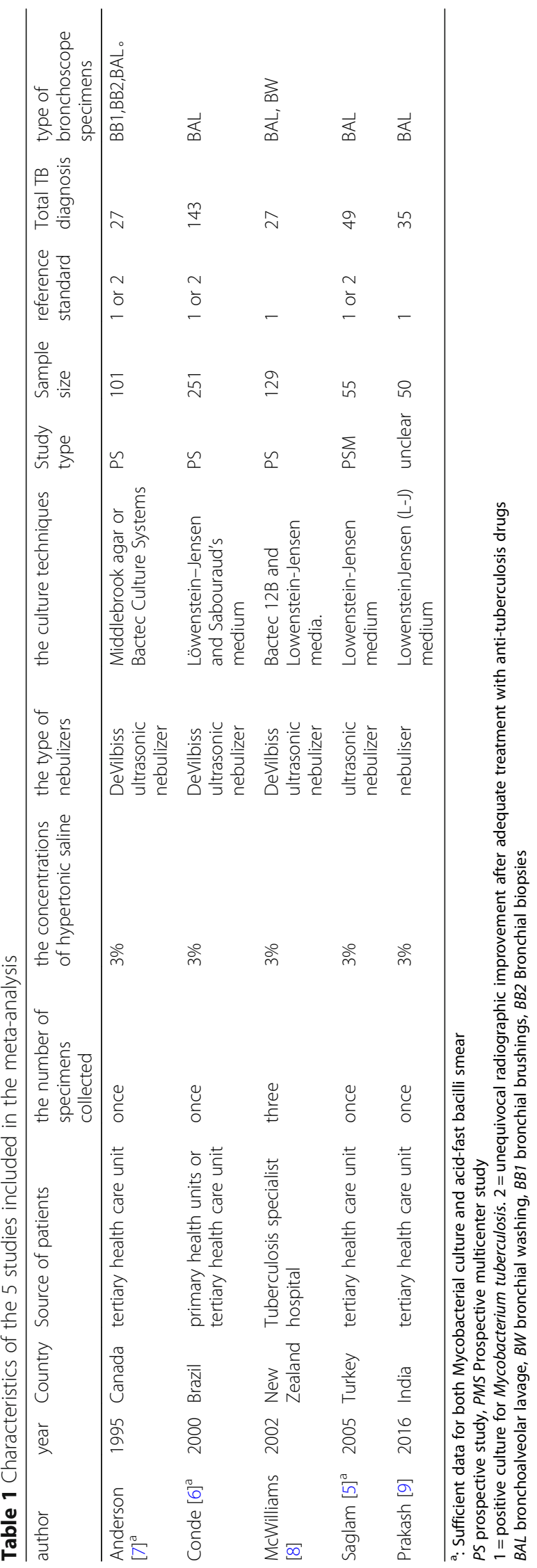




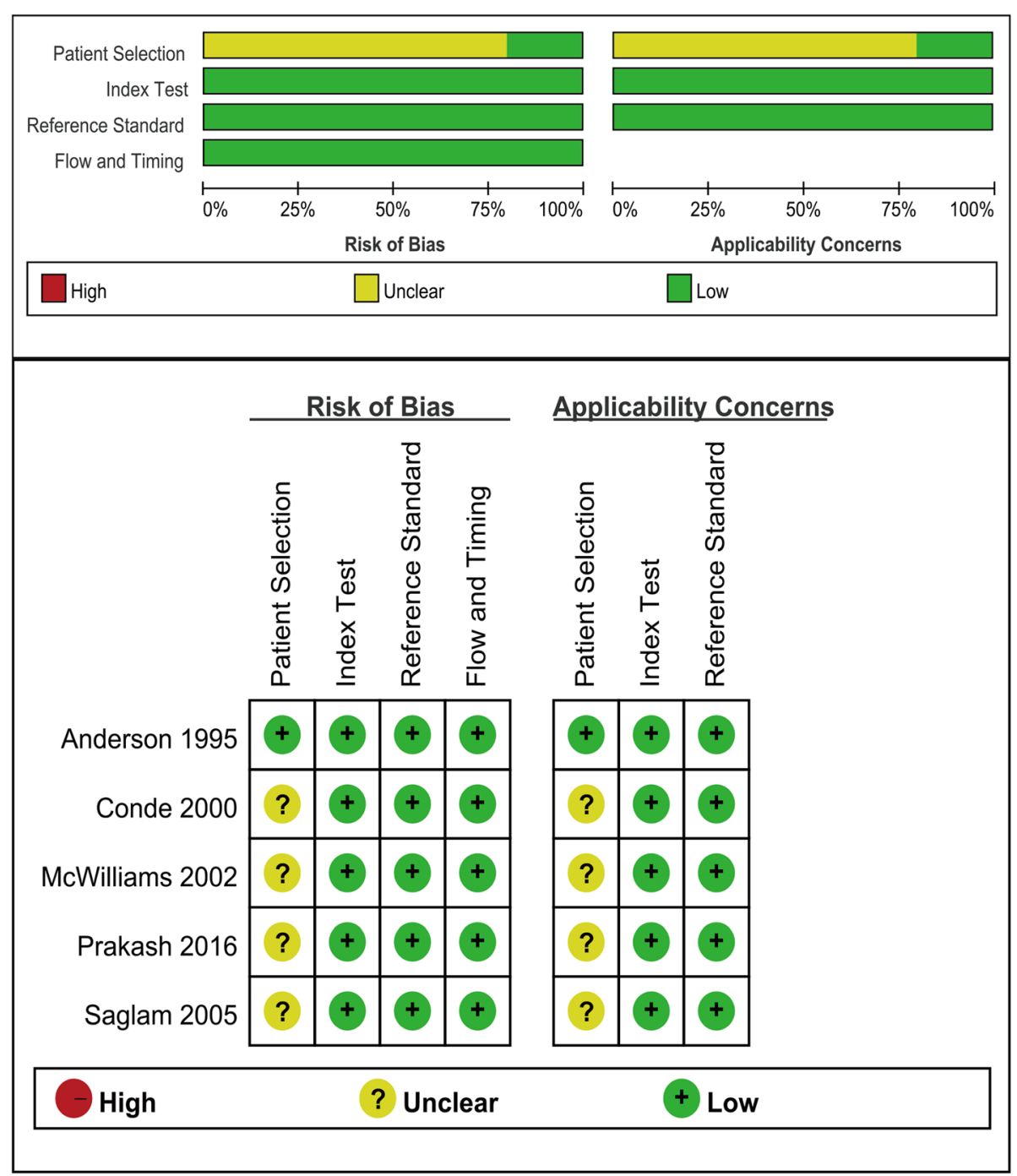

Fig. 2 Quality assessment of the included studies (QUADAS-2)

\section{Quantitative synthesis of mycobacterial culture}

The Spearman correlation coefficient and $P$ value of sputum induction were -0.300 and 0.624 respectively, while the Spearman correlation coefficient and $P$ value of bronchoscopy were 0.000 and 1.000 , both of which indicated that there was no significant threshold effect.
There was little heterogeneity among studies and the parameters of included studies could be pooled. There was no significant heterogeneity either in sputum induction studies (Cochran-Q of DOR $=5.55 ; p=0.235$ ) or in bronchoscopy studies (Cochran-Q of DOR = $2.71 ; p=0.608)$. Therefore the SEN, SPE, PLR, NLR

Table 2 Pooled results of sputum induction and bronchoscopy mycobacterial culture

\begin{tabular}{llllll}
\hline & Pooled SEN & Pooled SPE & Pooled + LR & Pooled -LR & Pooled DOR \\
$(95 \% \mathrm{Cl})$ & $(95 \% \mathrm{Cl})$ & $(95 \% \mathrm{Cl})$ & 57.57 & 0.26 & 280.08 \\
\hline sputum induction & 0.72 & 1.00 & $(16.89-196.30)$ & $(0.17-0.42)$ & $(58.93-1331.3)$ \\
Broncho-scopy & $(0.66-0.77)$ & $(0.99-1.00)$ & 51.02 & 0.33 & 166.43 \\
& 0.70 & 1.00 & $(14.93-174.32)$ & $(0.26-0.42)$ & $(45.81-604.61)$ \\
\hline
\end{tabular}




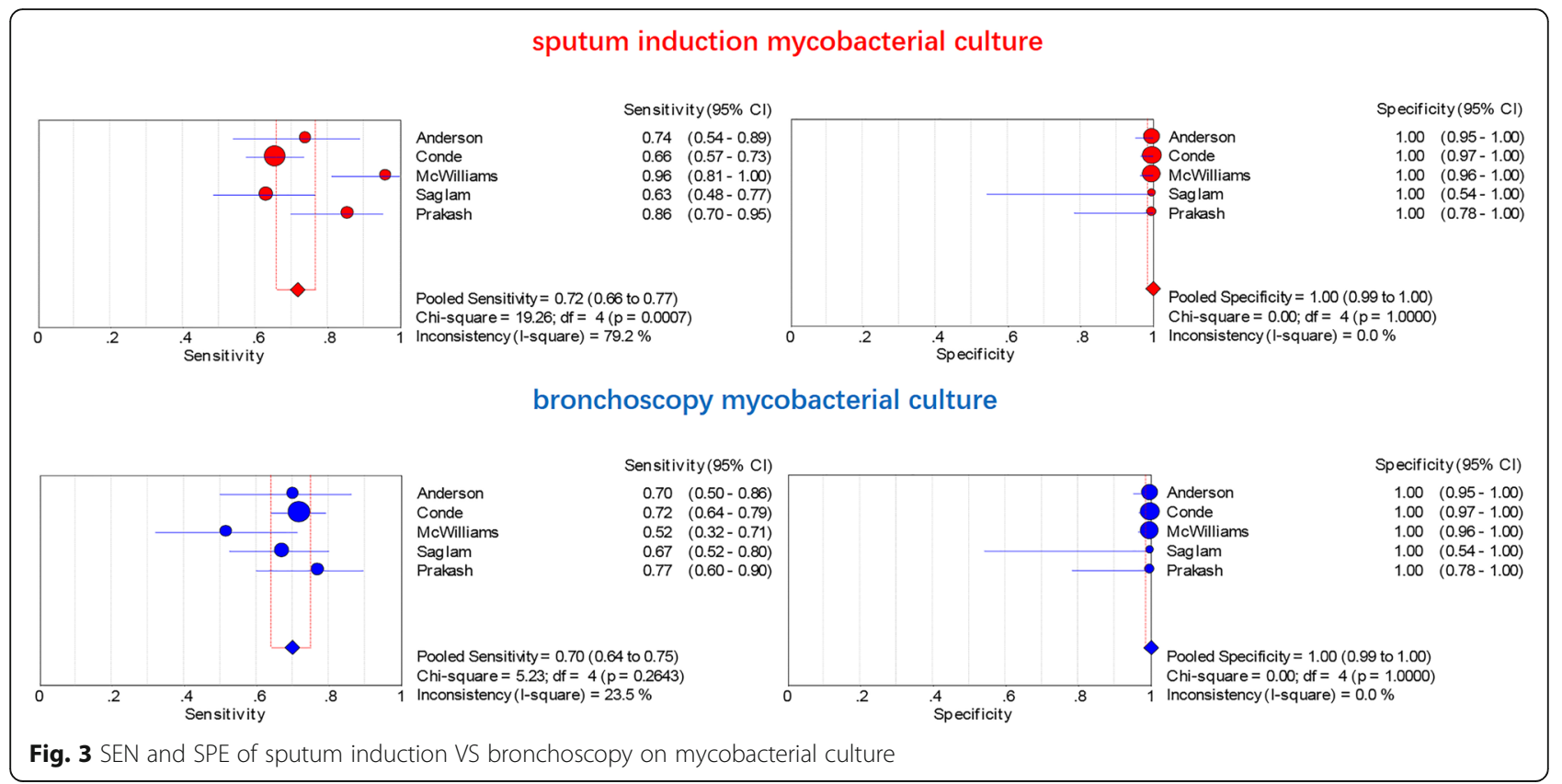

and DOR outcomes were pooled. In terms of mycobacterial culture, the SEN, SPE, PLR, NLR and DOR of sputum induction were $0.72(95 \% \mathrm{CI} \quad 0.66-0.77)$, $1.00(95 \%$ CI $\quad 0.99-1.00), \quad 57.57(95 \%$ CI $16.89-196.30)$, $0.26(95 \% \mathrm{CI} \quad 0.17-0.42)$ and $280.08(95 \% \mathrm{CI} 58.93-$ 1331.3) respectively, whereas the SEN, SPE, PLR, NLR and DOR of bronchoscopy were $0.70(95 \% \mathrm{CI}$ 0.64-
$0.75), \quad 1.00(95 \% \mathrm{CI} \quad 0.99-1.00), \quad 51.02(95 \% \mathrm{CI} \quad 14.93-$ $174.32), \quad 0.33(95 \% \mathrm{CI} \quad 0.26-0.42)$ and $166.43(95 \% \mathrm{CI}$ 45.81-604.61) respectively(Table. 2, Fig. 3). Sputum induction had a similar AUC (0.9564, SE $=0.0749)$ with bronchoscopy $(0.8618, \mathrm{SE}=0.1652) \quad(P$ value for difference of AUC between sputum induction and bronchoscopy was 0.602 Fig. 4.a).
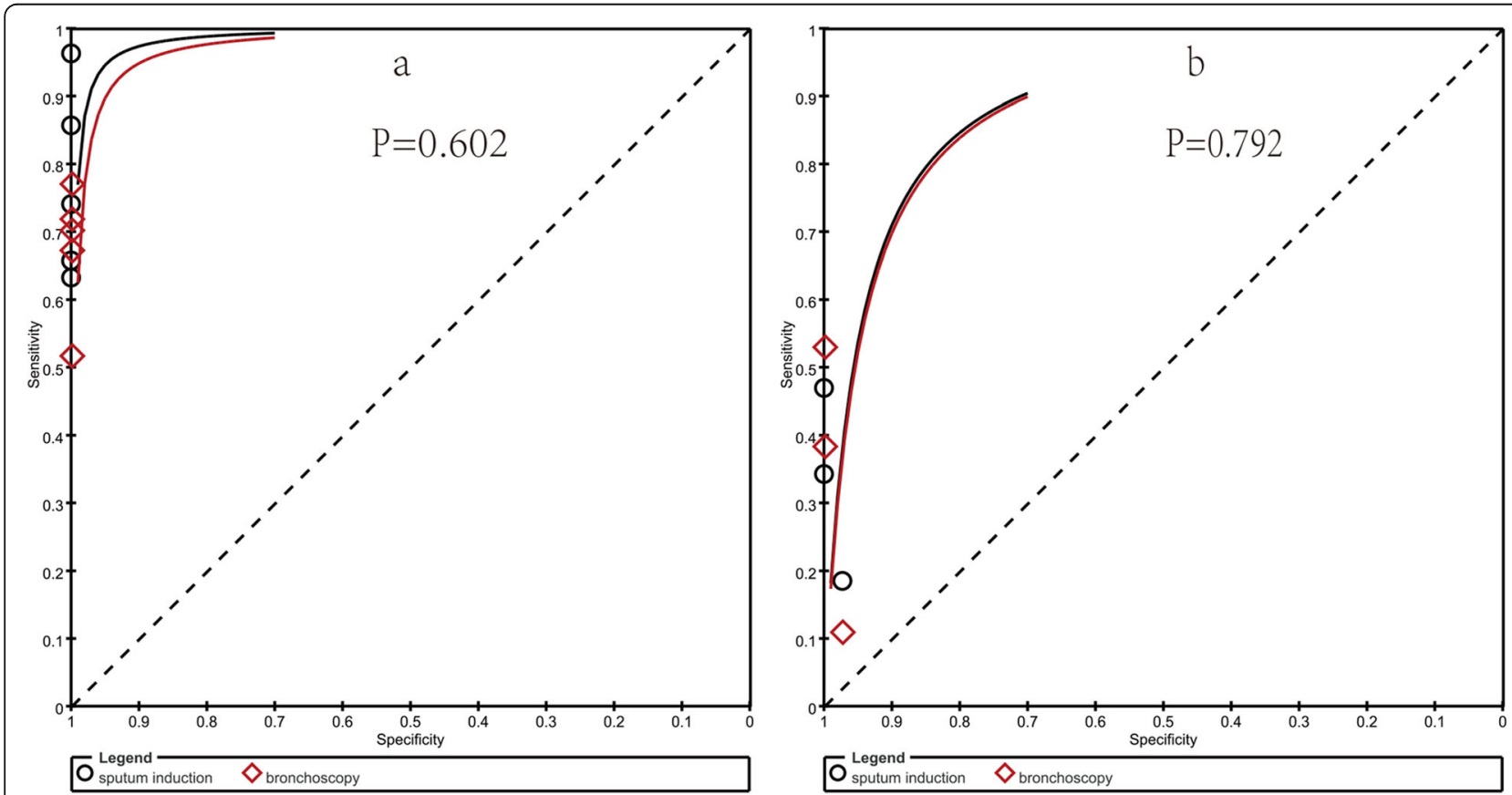

Fig. 4 ROC of sputum induction VS bronchoscopy on mycobacterial culture(a) and acid-fast bacilli smear(b) 


\section{Quantitative synthesis of acid-fast bacilli smear}

Only three articles had enough data on acid-fast bacilli smear [5-7]. The Spearman correlation coefficient and $P$ value of sputum induction (Spearman correlation coefficient: 0.500, $p$-value $=0.667)$ were same to bronchoscopy, both of which indicated that there was no significant threshold effect. There was no significant heterogeneity either in the sputum induction studies (Cochran-Q of DOR $=3.72 ; p=0.156$ ) or the bronchoscopy studies (Cochran-Q of DOR $=5.84 ; p=0.054$ ). The SEN, SPE, PLR, NLR and DOR outcomes were pooled. The SEN, SPE, PLR, NLR and DOR of sputum induction were $0.35(95 \% \mathrm{CI}, \quad 0.29-0.42), \quad 0.99(95 \% \mathrm{CI}, \quad 0.96-1.00)$, $12.72(95 \% \mathrm{CI}, \quad 2.14-75.76), \quad 0.70(95 \% \mathrm{CI}, \quad 0.57-0.85)$ and 18.78(95\%CI, 2.79-126.52) respectively, whereas the SEN, SPE, PLR, NLR and DOR of bronchoscopy were $0.38(95 \% \mathrm{CI}$, 0.32-0.45), 0.99(95\%CI, 0.96-1.00), 11.92(95\%CI, $1.27-$ 112.26), $0.67(95 \% \mathrm{CI}, 0.48-0.95)$ and $18.22(95 \% \mathrm{CI}, 1.55-$ 214.55) respectively(Additional file 1: Appendix S2). There was no significant difference on AUC between sputum induction (0.6016) and bronchoscopy $(0.8163 ; p$ value for difference of AUC between them was 0.792 Fig. 4.b).

\section{Mycobacterial culture versus acid-fast bacilli smear of sputum induction in the diagnosis of pulmonary tuberculosis}

In the three articles mentioned above [5-7], the mycobacterial culture was compared with acid-fast bacilli smear of sputum induction in the diagnosis of pulmonary tuberculosis. The SEN and SPE of mycobacterial culture were $0.66(95 \% \mathrm{CI}, 0.60-0.72)$ and $0.99(95 \% \mathrm{CI}, 0.98-$ 1.00) respectively, whereas the SEN and SPE of acid-fast bacilli smear were $0.35(95 \% \mathrm{CI}, 0.29-0.42)$ and 0.99(95\%CI, 0.96-1.00) respectively. The SEN of mycobacterial culture was higher than acid-fast bacilli smear $(p<0.001)$.

\section{Sensitivity analysis}

The combined AUC results were not materially altered after we sequentially excluded each study (Additional file 1: Appendix S3), suggesting that the results were not excessively dependent on a certain study.

\section{Publication bias}

The Deeks' funnel plot asymmetry test indicated no statistical evidence of publication bias in the sputum induction studies $(P=0.55)$. (Additional file 1: Appendix S4).

\section{Discussion}

Diagnosis of tuberculosis is still a challenge for those sputum smear negative pulmonary tuberculosis. The purpose of this meta-analysis was to compare the diagnosis value of sputum induction and bronchoscopy in the diagnosis of sputum smear-negative pulmonary tuberculosis. We found that sputum induction had similar diagnostic performance with bronchoscopy in the diagnosis of sputum smear-negative pulmonary tuberculosis, both in terms of acid-fast bacilli smear and mycobacterial culture.

As is well known, bronchoscopy is an important examination for obtaining high quality respiratory specimens in pulmonary infectious disease and also recommended for the diagnosis of pulmonary tuberculosis $[10,15,16]$. Nevertheless, this technique is invasive, poorly tolerated and costly compared with sputum induction. Additionally, bronchoscopy is not easily available in resourcelimited areas and it is not suitable in settings when a large quantity of people need to be evaluated.

Sputum induction is an uncomplicated, safe, cheap, and effective method for the diagnosis of pulmonary tuberculosis, which make it particularly suitable for being used in resource-limited settings [4, 17, 18]. Several studies have compared detection rates of sputum induction with bronchoscopy in tuberculosis cases. The results are variable and the sample size is relatively small $[5,9,19]$. Our meta-analysis showed that sputum induction had a similar overall diagnostic accuracy with bronchoscopy in sputum smear-negative pulmonary tuberculosis. Meanwhile, a prospective multicenter study showed that repeated induced sputum specimens could improve the diagnostic yield of pulmonary tuberculosis and it was not desirable to exclude the diagnosis of tuberculosis through a single specimen [19], which was in agreement with the recommendations of treatment of tuberculosis guidelines published by WHO [20]. Considering all of these, we suggest that for patients with suspected pulmonary tuberculosis who are smear-negative for acid-fast bacilli, sputum induction rather than bronchoscopy should be recommended as the initial method, which is in accordance with the guideline [10].

We compared the diagnostic yield of acid-fast bacilli smear with mycobacterial culture of sputum induction in pulmonary tuberculosis, and found that the SEN in mycobacterial culture was higher than in acid-fast bacilli smear, which is consistent with the previous study by Monkongdee et al. [21]. Our results are also in line with the guidelines for pulmonary tuberculosis diagnosis published by WHO [22]. So patients with suspected pulmonary tuberculosis who are acid-fast bacilli smear negative should undergo mycobacterial culture to increase the diagnostic yield of tuberculosis.

The limitations in our meta-analysis are as follows. First, the number of included studies was not large. This is because we only accepted studies that used sputum induction and bronchoscopy for detection of tuberculosis within the same population, which is also an advantage of this study because of the small heterogeneity. Second, since patients came from different places (some from primary health units and some from tertiary health care 
unit), the pretest probabilities of diagnosis of tuberculosis were different. Patients from tertiary health care unit had a higher pretest probability than those from primary health units, which could lead to potential heterogeneity. Third, the culture techniques in the different studies were different, but they were same for the culture of the two specimens (sputum induction and bronchoscopy specimen) in each study. So it didn't significantly affect the heterogeneity of this study. Fourth, we only compared the diagnostic value of acidfast bacilli smear or mycobacterial culture of sputum induction or bronchoscopy in tuberculosis, but were unable to compare the acid-fast bacilli smear joint mycobacterial culture of sputum induction or bronchoscopy, as there were no sufficient data of this topic for statistical analysis.

\section{Conclusions}

Sputum induction has similar sensitivity, specificity and overall accuracy compared to bronchoscopy-obtained specimens in diagnosing for sputum smear-negative pulmonary tuberculosis. Meanwhile, mycobacterial culture has a higher sensitivity than acid-fast bacilli smear in diagnosing for sputum smear negative tuberculosis.

\section{Supplementary information}

Supplementary information accompanies this paper at https://doi.org/10. 1186/s12890-020-01192-w.

Additional file 1: Appendix S1. Full search terms used in the literature search of Embase. Appendix S2. Pooled results of sputum induction and bronchoscopy acid-fast bacilli smear. Appendix S3. Sensitivity analysis. Appendix S4. Publication bias.

\section{Abbreviations}

SEN: Sensitivities; SPE: Specificities; PLR: Positive likelihood ratio; NLR: Negative likelihood ratio; DOR: Diagnostic odds ratio; Cl: Confidence interval; AUCs: Areas under the curves; WHO: World Health Organization; PRISMA: The Preferred Reporting Items for Systematic Reviews and MetaAnalyses; TB: Tuberculosis; TP: True positive; FP: False positive; TN: True negative; FN: False negative; QUADAS-2: The Quality Assessment of Diagnostic Accuracy Studies; SE: Standard Error

\section{Acknowledgements}

The authors would like to thank the Fujian Provincial Health Commission and Xiamen Municipal Health Commission for their support in this research.

\section{Consent to publication}

Not applicable.

\section{Authors' contributions}

SYH designed the study and revised the manuscript, LW and LYH conducted literature search, data analysis and drafted the manuscript. LZB and WWY conducted data analysis. The authors read and approved the final manuscript.

\section{Funding}

This study was supported by Youth Scientific Research Project (2016-2-64) of Fujian Provincial Health Commission and Xiamen Municipal Health Commission in China. The funders had no role in study design, data collection and analysis, decision to publish, or preparation of the manuscript.

\section{Availability of data and materials}

The dataset used and/or analyzed during the current study will be available from the corresponding author on a reasonable request after the final result is published in a journal.

\section{Ethics approval and consent to participate}

These were waived as this study was only based on the summary results of previously published articles and individual patient data was not obtained or accessed.

\section{Competing interests}

All authors declare no conflict of interest here.

\section{Author details}

${ }^{1}$ Department of Pulmonary and Critical Care Medicine, the First Affiliated Hospital of Xiamen University, Xiamen 361001, China. ${ }^{2}$ Epidemiology Research Unit, the First Affiliated Hospital of Xiamen University, Xiamen, China.

Received: 24 October 2019 Accepted: 19 May 2020

Published online: 25 May 2020

\section{References}

1. WHO. Global tuberculosis report 2018 [Available from: Available: http://www. who.int/tb/ publications/global_report/en. accessed on date 19 May.2019.

2. Strumpf IJ, Tsang AY, Sayre JW. Re-evaluation of sputum staining for the diagnosis of pulmonary tuberculosis. Am Rev Respir Dis. 1979;119(4):599-602.

3. Murray PR, Elmore C, Krogstad DJ. The acid-fast stain: a specific and predictive test for mycobacterial disease. Ann Intern Med. 1980;92(4):512-3.

4. Gonzalez-Angulo Y, Wiysonge CS, Geldenhuys H, Hanekom W, Mahomed H, Hussey $\mathrm{G}$, et al. Sputum induction for the diagnosis of pulmonary tuberculosis: a systematic review and meta-analysis. Eur J Clin Microbiol Infect Dis. 2012;31(7):1619-30.

5. Saglam L, Akgun M, Aktas E. Usefulness of induced sputum and fibreoptic bronchoscopy specimens in the diagnosis of pulmonary tuberculosis. J Int Med Res. 2005;33(2):260-5.

6. Conde MB, Soares SL, Mello FC, Rezende VM, Almeida LL, Reingold AL, et al. Comparison of sputum induction with fiberoptic bronchoscopy in the diagnosis of tuberculosis: experience at an acquired immune deficiency syndrome reference center in Rio de Janeiro, Brazil. Am J Respir Crit Care Med. 2000;162(6):2238-40.

7. Anderson C, Inhaber N, Menzies D. Comparison of sputum induction with fiber-optic bronchoscopy in the diagnosis of tuberculosis. Am J Respir Crit Care Med. 1995;152(5 Pt 1):1570-4.

8. McWilliams T, Wells AU, Harrison AC, Lindstrom S, Cameron RJ, Foskin E. Induced sputum and bronchoscopy in the diagnosis of pulmonary tuberculosis. Thorax. 2002;57(12):1010-4.

9. Prakash P, Agarwal P, Gupta A, Gupta E, Dasgupta A. Comparison of induced sputum and Bronchoalveolar lavage fluid examination in the diagnosis of sputum negative pulmonary tuberculosis. Indian J Chest Dis Allied Sci. 2016;58(3):173-5.

10. Lewinsohn DM, Leonard MK, Lobue PA, Cohn DL, Daley CL, Desmond E, et al. Official American Thoracic Society/Infectious Diseases Society of America/Centers for Disease Control and Prevention clinical practice guidelines: diagnosis of tuberculosis in adults and children. Clin Infect Dis. 2017;64(2):e1-e33.

11. Whiting PF, Rutjes AW, Westwood ME, Mallett S, Deeks JJ, Reitsma JB, et al. QUADAS-2: a revised tool for the quality assessment of diagnostic accuracy studies. Ann Intern Med. 2011:155(8):529-36.

12. Higgins JPT, Thompson SG. Quantifying heterogeneity in a meta-analysis. Stat Med. 2002;21(11):1539-58

13. Song F, Khan KS, Dinnes J, Sutton AJ. Asymmetric funnel plots and publication bias in meta-analyses of diagnostic accuracy. Int J Epidemiol. 2002;31(1):88-95.

14. Team RC. R: a language and environment for statistical computing. Vienna: R Foundation for Statistical Computing; 2019.

15. De Gracia J, Curull V, Vidal R, Morell F. Bronchoscopy with bronchoalveolar lavage in the diagnosis of pulmonary tuberculosis. Chest. 1992;101(1):292.

16. Kennedy DJ, Lewis WP, Barnes PF. Yield of bronchoscopy for the diagnosis of tuberculosis in patients with human immunodeficiency virus infection. Chest. 1992;102(4):1040-4. 
17. Hepple P, Ford N, McNerney R. Microscopy compared to culture for the diagnosis of tuberculosis in induced sputum samples: a systematic review. Int J Tuberc Lung Dis. 2012;16(5):579-88.

18. Morse M, Kessler J, Albrecht S, Kim R, Thakur R, Nthobatsang R, et al. Induced sputum improves the diagnosis of pulmonary tuberculosis in hospitalized patients in Gaborone, Botswana. Int J Tuberc Lung Dis. 2008; 12(11):1279-85.

19. Schoch OD, Rieder P, Tueller C, Altpeter E, Zellweger JP, Rieder HL, et al. Diagnostic yield of sputum, induced sputum, and bronchoscopy after radiologic tuberculosis screening. Am J Respir Crit Care Med. 2007;175(1): 80-6.

20. WHO Guidelines Approved by the Guidelines Review Committee. Treatment of Tuberculosis: Guidelines (the fourth edition). Geneva: WHO; 2010.

21. Monkongdee P, McCarthy KD, Cain KP, Tasaneeyapan T, Nguyen HD, Nguyen TN, et al. Yield of acid-fast smear and mycobacterial culture for tuberculosis diagnosis in people with human immunodeficiency virus. Am J Respir Crit Care Med. 2009;180(9):903-8.

22. World Health Organization. Improving the diagnosis and treatment of smear-negative pulmonary and extrapulmonary tuberculosis among adults and adolescents: recommendations for HIV-prevalent and resourceconstrained settings. Geneva: WHO; 2006.

\section{Publisher's Note}

Springer Nature remains neutral with regard to jurisdictional claims in published maps and institutional affiliations.

Ready to submit your research? Choose BMC and benefit from:

- fast, convenient online submission

- thorough peer review by experienced researchers in your field

- rapid publication on acceptance

- support for research data, including large and complex data types

- gold Open Access which fosters wider collaboration and increased citations

- maximum visibility for your research: over $100 \mathrm{M}$ website views per year

At $\mathrm{BMC}$, research is always in progress.

Learn more biomedcentral.com/submissions 